\title{
The Political Districting Problem: A Survey*
}

\author{
Attila Tasnádi \\ Department of Mathematics, Corvinus University of Budapest, H - 1093 Budapest, \\ Fơvám tér 8, Hungary, attila.tasnadi@uni-corvinus.hu
}

November 2009

Appeared in Society and Economy 33(2011), 543-554.

(C)Akadémia Kiadó*

\begin{abstract}
Computer scientists and social scientists consider the political districting problem from different viewpoints. This paper gives an overview of both strands of the literature on districting in which the connections and the differences between the two approaches are highlighted.
\end{abstract}

Keywords: Political districting, gerrymandering.

JEL Classification Number: D72

\footnotetext{
${ }^{*}$ The author gratefully acknowledges financial support from the Corvinus University of Budapest through its Research Excellence Fellowship.

*The original article is available at http://dx.doi.org/10.1556/SocEc.2011.0001
} 


\section{Introduction}

Districting plays a crucial role in all electoral systems endowed with single member districts. The underlying problem is that an actual redistricting, which has to be carried out in order to prevent malapportionment (i.e. unequal representation of citizens), may favor a specific party. The resulting bias could be caused by an ex ante unbiased districting procedure (where by ex ante unbiasedness we mean that the bias and its extent towards a party is not known prior to the employment of the given districting procedure) or consciously by the body in charge of the redistricting process. The latter goes back to 1812 when Elbrige Gerry Governor of Massachusetts reluctantly signed a redistricting plan favoring Jeffersonian democrats into law. The word gerrymandering, standing for the activity of carrying out a redistricting in favor of a certain party, was coined from Gerry's name and the salamander like looking districts of Massachusetts in 1812. Since then gerrymandering is a hot issue in the United States and is still happening because in most US states the legislature has primary responsibility for creating a redistricting plan, often subject to approval by the state governor. ${ }^{1}$ Other countries, like Hungary, have not carried out a redistricting at all because the parties fear that they may be harmed by the new districting. Of course, lack of redistricting over a long time leads to huge inequalities in district sizes.

The paper focuses on the computer science, the economics and the political science literature; and thus, it does not present the large literature on legal issues and court cases. Originally, it was hoped in the mid of the 20th century that through the use of modern computers the problem of gerrymandering could be overcome since a computer program just using data on voters distributions without any statistical information on voters preferences should not be biased towards a party (e.g. Vickrey, 1961). Not surprisingly this view was too optimistic because a computer programmer may write a program favoring a party in a disguised form and because there are no precise measures of ex ante unbiasedness. Even if there is a good measure for ex ante unbiasedness, parties should fear ex post bias. For more on the effects of the increasing role played by computers in determining districting plans from a historical perspective consult Altman (2005). The economics literature tries to determine party strategies, the effects of gerrymandering on government policy and social welfare. For instance, one may pose the question whether gerrymandering is harmful at all from an economical point of view. Though there is no clear borderline between the economics and the political science literature, the latter is more interested, for example, in the effects of redistricting on the emerging party structure, on voters' behavior (participation rate), on the competitiveness of districts and on the rights of minorities.

The remainder of the paper is organized as follows. The next section presents a survey of the computational literature in which determining ex ante unbiased districtings and the computational complexity of the districting problem are considered. Section 3 deals with the social science literature on districting. Section 4 offers some concluding remarks.

\footnotetext{
${ }^{1}$ For more details on redistricting practice, also outside the US, see e.g. http://en.wikipedia.org/wiki/Redistricting (accessed: 11/10/2009).
} 


\section{The computational approach to districting}

A solution of the political districting problem partitions the state into territorial units (districts). Ex ante unbiased districtings usually require the following conditions to be satisfied by a districting.

- Population equality requires that the populations of the districts should be as equal as possible. This is a strong condition in the US, where only small district size deviations are tolerated by court, whereas in many European countries larger deviations (for instance, a 15\% deviation in population from the average district population) are allowed.

- Contiguity means that a district cannot be disconnected; that is, any two points within the district can be connected through a path remaining in that district.

- Compactness prescribes that the shapes of the districts should not be to long and thin. Thus, rectangular and circular districts should be preferred.

Since the above three criteria are independent of the voters' party preferences it is widely believed that an algorithm meeting these three criteria is in some sense unbiased. While population equality is a precise condition, contiguity and compactness are ambiguous. ${ }^{2}$ In fact, the Supreme Court always rejected appeals claiming unlawful gerrymandering based on loosely connected or oddly looking districts, since it could be the case that population equality and minority rights did not allow for a more contiguous and more compact districting. However, most of the computational literature focused on algorithms finding ex ante unbiased solutions.

\subsection{Early algorithms determining ex ante unbiased districtings}

Vickrey (1961) pointed out by example that reasonable districtings could produce completely different outcomes in the number of winning districts. Therefore, he propagated that the human element should be removed from the process and random elements should be integrated into a procedure so that the actual districting plan cannot be predicted. Informally, he sketched a procedure not guaranteeing a contiguous and compact districting. The main purpose of his paper was to show that mechanical rules without human influence could solve the political districting problem. However, his proposal was quite rudimentary and impractical for real life problems.

Hess et al. (1965) developed an ex ante unbiased method to solve the legislative districting problem of Delaware by observing that the political districting problem is analogous to the so-called 'warehouse-location' problem. The legislative districts (LD) were constructed from inseparable smaller units called enumeration districts (ED). Their procedure (1) needed a heuristic guess for the initial district centers, (2) a transportation algorithm was used to assign EDs population equally to district centers, (3) an adjustment procedure ensured that each ED was contained entirely in one LD, (4) new district centers were determined from the obtained LDs, (5) go to step 2 if the change of district centers is too large. The main shortcomings of their procedure were

\footnotetext{
${ }^{2}$ For recent advancements on measuring district compactness see Fryer and Holden (2007) and Chambers and Miller (2009).
} 
that its result depended on the initial guess of district centers and that its convergence was not proven. However, one of their redistricting plans was used for legislative redistricting in Delaware.

Kagel's (1966) procedure obtained a districting as a solution of a weighted optimization problem, which simultaneously strives for population equality and for district 'compactness'. His procedure starts from an initial districting (actual districting) and sequentially tries to improve the object function by checking moves of an ED from its current LD to another neighboring LD and by checking bilateral exchanges of EDs between two LDs. The main shortcomings of his procedure are that it might be trapped in a local optimum and that it depends very much on the initial districting.

Garfinkel and Nemhauser (1970) provided the first algorithm finding all ex ante unbiased districtings. Therefore, a more detailed description of their algorithm is given here. $M$ districts have to be formed from $N$ given population units with respective populations $p_{1}, \ldots, p_{N}$. Let $\bar{p}=\sum_{i=1}^{N} p_{i} / M$ be the mean population. The elements of a zero-one matrix $A=\left(a_{i j}\right)_{i=1, j=1}^{N, M}$ indicate whether population unit $i$ is assigned to district $j$. Hence, the population of district $j$ equals $P(j)=\sum_{i=1}^{N} a_{i j} p_{i}$. Population equality is captured by condition

$$
c_{j}=|P(j)-\bar{p}| \leq \alpha \bar{p},
$$

where $\alpha \in[0,1]$ is the maximum relative deviation of district population from the average district population. An adjacency matrix $B=\left(b_{i j}\right)_{i=1, j=1}^{N, N}$ indicates whether two population units have a common boundary. A solution satisfies contiguity if the resulting districts are connected in the graph given by the adjacency matrix $B$. The compactness of district $j$ is determined by $c_{j}^{\prime}=d_{j}^{2} / A(j)$, where $d_{j}$ equals the largest distance between to population units within district $j$ and $A(j)$ denotes the area of district $j$. An upper bound on district compactness is given by parameter $\beta$. The zero-one matrix $A$ specifies a feasible districting if it satisfies the population equality constraints (2.1), its associated graph is connected and satisfies the connectedness condition. The objective is to determine the feasible districtings having the smallest maximum population inequality $\left(\min \max c_{j}\right)$. The algorithm determines in the first phase the set of all feasible districts and in the second the optimal districtings. The employed enumeration technique ensures that the algorithm finds a solution if the set of feasible districtings is non-empty. However, the required number of computations explodes as $N$ increases. Clearly, having smaller population units produces better districtings, but increases the number of computations.

\subsection{The computational complexity of districting problems}

Nagel (1972) already pointed out in an early survey that automated redistricting has its computational limitations by considering the available programs of his time. Hence, one could only speak about computer aided redistricting in which the computer merely serves as a tabulating device.

The computational complexity of determining an ex ante unbiased districting was identified by Altman (1997). In particular, he established that meeting the three basic criteria of ex ante unbiasedness is an NP-hard problem. In addition, he demonstrated that maximizing the number of competitive districts is also NP-hard.

Nagel (1972) had already mentioned that a party with an overall support of $40 \%$ should receive, for instance, at least $15 \%$ of seats. Hence, ex ante unbiasedness is 
not really a good criterion for a satisfactory algorithm. Recently, Puppe and Tasnádi (2008) showed that achieving ex post unbiasedness, which requires that the number of winning districts of a party should be up to integer rounding proportional to its share in votes, is an NP-complete problem. Puppe and Tasnádi (2009) established in a follow up work that determining an optimal gerrymandering is also NP-complete.

\subsection{Recent districting algorithms}

The computational complexity of the political districting problems led to alternative approaches to determine ex ante unbiased districtings. A short and non-complete list of contributions follows.

Bação et al. (2005) employed genetic algorithms in which the encoding consists of a string of geographic information of districts and the fitness function follows from the criteria of ex ante unbiasedness. They also determined a districting of Lisbon city in Portugal. Chou and Li (2006) applied the $q$-state Potts model of statistical physics by which they determined the legislative districts for Taipei city in Taiwan. Various heuristics have been introduced by Mehrotra et al. (1998), Bozkaya et al. (2003), Ricca and Simeone (2008) and Ricca et al. (2008).

\section{The social science literature on districting}

This section contains many interesting contributions to the political districting problem appearing in the economics and the political science literature. Since the social science literature on political districting is very large, only a selective survey of empirical and theoretical works follows.

Owen and Grofman (1988) determined the optimal gerrymandering for a risk-averse party controlling the redistricting process in a two-party system with only singlemember districts in an uncertain world. The optimal solution has the characteristic that the gerrymanderer creates on the one side districts with equal and comforting winning probabilities and on the other side districts full with its opponent supporters; therefore, this solution is called bimodal by many others.

Gelman and King (1994) surprisingly found that redistricting increases the electoral responsiveness (that is, how legislative composition reacts to voters' preferences). Another less surprising but important result of their paper is that redistricting, no matter how biased it is, is better than having no redistricting at all. They obtained these results through a regression model using US election results from 1968 to 1988 containing 267 statewide elections and 29,679 district-level elections.

Sherstyuk (1998) combined the cake-cutting literature with the optimal gerrymandering problem. As Owen and Grofman (1988) she assumed that a party is in control of the redistricting process but she takes geographical constraints into consideration and allows for more general distributions of voters' characteristics. She identifies objective functions and conditions under which the optimal gerrymandering solution remains bimodal. It should be mentioned that her results are non-constructive because they rely on Lyapunov's existence theorem. District compactness does not appear in her geographical conditions and contiguity is achieved through arbitrarily thin paths.

Gilligan and Matsusaka (1999) investigates partisan bias empirically and theoretically in function of the number of voters and the number of single-member districts. 
They find that within a jurisdiction an increase in population increases partisan bias, while an increase in districts decreases partisan bias under optimal gerrymandering. Hence, an increase in the overall population should be followed by an increase in the number of seats since otherwise partisan bias would inevitably increase. Their findings were tested on data from U.S. elections over the period 1950-1994. In another paper Gilligan and Matsusaka (2006) develops a simple theory of redistricting based on the median voter theorem. The policy outcome in their model is determined by the median legislator, which should equal the policy choice of the median voter in the absence of bias. Since the legislators are in fact the median voters of their own districts, the median legislator usually does not equal the median voter in case of partisan districting. One of their main finding is that the resulting policy bias favors non-majority groups. Another interesting result is that random districting does not eliminate policy bias on average.

Shotts (2002) develops a three-stage game-theoretic model in which, first, redistricting is carried out in each state by a gerrymanderer, second, the voters determine the composition of the House of Representatives, and third, the policy choice is made. The main point is that since racial gerrymandering is unlawful and liberal voters (leftist) are more susceptible to racial gerrymandering, conservative gerrymanderers are more constrained than liberal gerrymanderers. Therefore, the final policy outcome is biased towards liberals contrary to what many political scientists believe. In an earlier paper Shotts (2001) focuses on the partisan instead of the policy effect of mandatory majority-minority districts (in which statewide minorities have a majority).

The seat-vote curve describes the relationship between the relative share in seats and the relative share in votes. In case of a proportional representation system the seat vote curve is linear. Two important measures related to seat-vote curves are bias and responsiveness: The former one measures the difference of the realized seat share of a party from its proportional seat share based on its vote share, while the latter measures the marginal effect of a change in a party's share of votes on its seat share. Coate and Knight (2007) characterize the socially optimal seat-vote curve and shows the existence of a districting producing an optimal seat-vote curve. They also develop a methodology for computing real-life seat-vote curves and consider welfare gains arising from an optimal districting. Their paper assumes that a districting authority tries to maximize social welfare in an environment of two parties (Democrats and Republicans) and of three categories of voters (Democrats, Republicans and Independents) whose ideological stance can be measured by a value between zero (ardent Democrat) and one (ardent Republican). The payoff of a citizen having ideology $x \in[0,1]$ is given by $\beta-\gamma\left(x-x^{\prime}\right)^{2}$, where $x^{\prime}$ is the ideology of its legislation, $\beta$ is its payoff if its ideology is fully matched by the legislation's ideology and $\gamma$ is a dissipation factor. The state has to be divided into $n$ equal districts without geographical constraints and a given districting determines social welfare by aggregating the payoffs of the individuals. As a result they obtained a linear optimal seat-vote curve with bias towards the party having the larger partisan base and responsiveness depending on swing voters (Independents). Since the socially optimal districting has been derived without considering only feasible districtings, ${ }^{3}$ Coate and Knight also give a condition for the implementability of the obtained socially optimal linear seat-vote curve.

From Coate and Knight (2007) independently Besley and Preston (2007) gave a

\footnotetext{
${ }^{3}$ Here feasibility does not mean geographic feasibility, but the sustainability of a technical assumption simplifying the derivation of the socially optimal seat-vote curve.
} 
similar micro foundation of seat-vote curves to determine how electoral districting effects policy choice. They obtain that more electoral bias leads to more extreme policy choices. In an empirical part they employ their model to local governments in England.

Chambers (2008) developed an axiomatic theory of political representation in which he examined the possibility of a gerrymandering-proof voting rule. In the first stage voters elect an alternative in each district by a non-specified voting rule and in the second stage the districts' outcomes are aggregated by the same voting rule to a nationwide outcome. Allowing that $n$ voters can be partitioned into $k$ districts without any kind of district size or geographical constraints, a gerrymandering-proof rule is either a pathological one (selects always the same alternative out of two) or has to allow for a set of countably infinite outcomes. Coming closer to the election of the House of Representatives by allowing only two alternatives to vote for and admitting any possible winning percentage of a party, under some technical conditions only quasiproportional rules (defined through so-called quasi-arithmetic means ${ }^{4}$ ) constitutes the set of gerrymandering-proof rules. Chambers (2009) establishes that under some natural technical conditions a voting rule is consistent if and only if it is a partial priority rule, where consistency means that the final outcome is independent of the employed partitioning of voters and a partial priority rule allows each voter to veto certain alternatives. To obtain the result one can restrict itself to equally sized districts. However, even Chamber's second paper does not impose any kind of geographical constraints.

Friedman and Holden (2008) consider the case in which one of the two parties in charge of the districting process faces a noisy signal of voter preferences. A finite number of equally sized districts have to be formed without geographical constraints. Voter's preferences are measured on a one-dimensional scale, where the most rightwing voter and the most left-wing voter are at the two ends. Thus, the paper can make suggestions on how a gerrymanderer should handle less or more loyal party voters in determining an optimal gerrymander. For instance, it has been shown that it does not payoff to concentrate one's most ardent opponents into the same district. Based on this result they conclude that partisan gerrymandering implies also racial gerrymandering, which is strictly forbidden by law, since major parts of African-Americans are ardent supporters of Democrats.

Gul and Pesendorfer (2010) analyze a game in which two parties carry out the redistricting within the territories under their legislative control and voters' types are unknown at the time of drawing the new district lines. Both parties try to maximize the probability of getting a majority in the House of Representatives. Assuming a continuum of voters and a continuum of districts they find, among many other interesting results, that both parties maximally segregate voters of unfavorable types, which is in line with common wisdom.

\section{Conclusion}

Large parts of the computational literature focused on determining ex ante unbiased districtings, which were determined by algorithms not using data on voters preferences. Because of the computational complexity of the problem there is on going research for

\footnotetext{
${ }^{4}$ The quasi-arithmetic mean of $x_{1}, \ldots, x_{n}$ is defined by $g^{-1}\left(\frac{\sum_{i=1}^{n} g\left(x_{i}\right)}{n}\right)$, where $g:[0,1] \rightarrow \mathbb{R}$ has to be a continuous, strictly increasing function.
} 
finding new and faster algorithms. All of the presented algorithms have been applied to real life districting problems and some of these results were used to solve actual districting problems. Ex ante unbiasedness is not an acceptable solution for parties since any given solution probably favors one of the parties. This might be one of the reasons why these algorithms have not been used widely. Clearly, neither parties nor independent juries are too happy about a random like distribution of seats. Though there are many computer programs searching for the optimal gerrymandering, the computer science literature has devoted less space to these algorithms probably because they do not solve the gerrymandering problem.

The social science literature escapes the computational complexity of the districting problem by considering simplified settings for which an optimal solution can be

determined. Based on these 'toy' models social scientists try to address qualitative questions like:

- What are the key features of an optimal gerrymander?

- How does gerrymandering effect policy choice, minorities and social welfare?

Though some of the answers to these questions could be empirically validated, it is not clear if neglecting geographical constraints like planarity, connectedness and contiguity, does not destroy some of the qualitative answers completely. May be models of bounded rationality could reduce the gap between the computational and social science literature.

\section{References}

[1] M. Altman, Is Automation the Answer? The Computational Complexity of Automated Redistricting. Rutgers Computer and Law Technology Journal 23(1) 81-142 (1997).

[2] M. Altman, K. Mac Donald, and M.P. McDonald, From Crayons to Computers: The Evolution of Computer Use in Redistricting. Social Science Computer Review 23(3) 334-346 (2005).

[3] F. Bação, V. Lobo and M. Painho, Applying Genetic Algorithms to zone Design. Soft Computing 9(5) 341-348 (2005).

[4] T. Besley and I. Preston, Electoral Bias and Public Choice: Theory and Evidence. Quarterly Journal of Economics 122(4) 1473-1510 (2007).

[5] B. Bozkaya, E. Erkut and G. Laporte, A Tabu Search Heuristic and Adaptive Memory Procedure for Political Districting. European Journal of Operational Research 144(1) 12-26 (2003).

[6] P.C. Chambers, Consistent Representative Democracy. Games and Economic Behavior 62(2), 348-363 (2008).

[7] P.C. Chambers, An Axiomatic Theory of Political Representation. Journal of Economic Theory, 144(1), 375-389 (2009).

[8] P.C. Chambers and A.D. Miller, A Measure of Bizarreness. Quarterly Journal of Political Science forthcoming (2009). 
[9] C-I. Chou and S.P. Li, Taming the Gerrymander-Statistical Physics Approach to Political Districting Problem. Physica A 369(2) 799-808 (2006).

[10] S. Coate and B. Knight, Socially Optimal Districting: A Theoretical and Empirical Exploration. Quarterly Journal of Economics 122(4) 1409-1471 (2007).

[11] J.N. Friedman and R.T. Holden, Optimal Gerrymandering: Sometimes Pack, But Never Crack. American Economic Review 98(1) 113-144 (2008).

[12] R.G. Fryer and R.T. Holden, Measuring the Compactness of Political Districting Plans. Mimeographed (2007).

[13] R.S. Garfinkel and G.L. Nemhauser, Optimal Political Districting by Implicit Enumeration Techniques. Management Science 16(8) 495-508 (1970).

[14] A. Gelman and G. King, Enhancing Democracy through Legislative Redistricting. American Political Science Review 88(3) 541-559 (1994).

[15] T.W. Gilligan and J.G. Matsusaka, Structural Constraints on Partisan Bias under the Efficient Gerrymander. Public Choice 100(1-2) 65-84 (1999).

[16] T.W. Gilligan and J.G. Matsusaka, Public Choice Principles of Redistricting. Public Choice 129(3-4) 381-398 (2006).

[17] R. Gul and W. Pesendorfer, Strategic Redistricting. American Economic Review forthcoming (2010).

[18] S.W. Hess, J.B Weaver, H.J. Siegfeldt, J.N. Whelan and P.A. Zitlau, Nonpartisan Political Redistricting by Computer. Operations Research 13(6) 998-1006 (1965).

[19] H.F. Kaiser, An Objective Method for Establishing Legislative Districts. Midwest Journal of Political Science 10(2) 200-213 (1966).

[20] A. Mehrotra, E.L. Johnson and G.L. Nemhauser, An Optimization Based Heuristic for Political Districting. Management Science 44(8) 1100-1114 (1998).

[21] S.S. Nagel, Computers \& the Law \& Politics of Redistricting. Polity 5(1) 77-93 (1972).

[22] G. Owen and B. Grofman, Optimal Partisan Gerrymandering. Political Geography Quarterly 7(1), 5-22 (1988).

[23] C. Puppe and A. Tasnádi, A Computational Approach to Districting. Mathematical Computer Modelling 48(9-10), 1455-1460 (2008).

[24] C. Puppe and A. Tasnádi, Optimal redistricting under geographical constraints: Why "pack and crack" does not work. Economics Letters 105(1), 93-96 (2009).

[25] F. Ricca and B. Simeone, Local Search Algorithms for Political Districting. Political Districting. European Journal of Operational Research 189(3) 1409-1426 (2008).

[26] F. Ricca, A. Scozzari and B. Simeone, Weighted Vornoi Region Algorithms for Political Districting. Mathematical Computer Modelling forthcoming (2008). 
[27] K. Sherstyuk, How to Gerrymander: A Formal Analysis. Public Choice 95(1-2) 27-49 (1998).

[28] K.W. Shotts, The Effect of Majority-Minority Mandates on Partisan Gerrymandering. American Journal of Political Science 45(1) 120-135 (2001).

[29] K.W. Shotts, Gerrymandering, Legislative Composition and National Policy Outcomes. American Journal of Political Science 46(2) 398-414 (2002).

[30] W. Vickrey, On the Prevention of Gerrymandering. Political Science Quarterly 76(1) 105-110 (1961). 